\title{
ИНФОРМАЦИОННЫЕ СИСТЕМЫ КОНТРОЛЯ КАЧЕСТВА ЗНАНИЙ
}

Бабешко В.Н.

Совершенствование образовательной системы связывают с необходимостью управления качеством образовательных услуг. Контроль качества знаний является важной частью ученого процесса. Проникновение компьютеров во все области деятельности меняет подходы и технологии, которые ранее в них применялись.

Ключевые слова: автоматизация; диагностика; качество; контроль; сетевой; система; технология; тестирование.

\section{INFORMATION SYSTEM QUALITY CONTROL KNOWLEDGE}

\section{Babeshko V.N.}

The development of the educational system is associated with the need to control the quality of educational services. Quality control knowledge is an important part of the scientific process. The penetration of computers into all areas of activities changing approaches and technologies that previously they were used.

Keywords: automation; diagnostics; quality control; network; system; technology; testing.

Потребители образовательных услуг все большое внимание уделяют их качеству, оно становится социально востребованной категорией. Проблема контроля качества знаний всегда была и 
остается крайне актуальной. Сегодня в педагогических исследованиях изучаются средства и способы повышения эффективности обучения и образования. Педагогический контроль является необходимой составляющей педагогического процесса и любой технологии обучения, в дистанционной он приобретает особо высокую значимость [1, с. 151].

В последнее время уделяется большое внимание разработке сетевых автоматизированных обучающих систем, позволяющих студенту в интерактивном режиме изучать и закреплять материал, а также проверять полученные знания. Положительные качества сетевых автоматизированных систем для компьютерного тестирования очевидны. Они позволяют освободить преподавателя от мало эффективной работы при проведении экзаменов и промежуточной оценке знаний в традиционном учебном процессе, а при обучении с использованием дистанционных технологий становятся основным средством контроля. Использование автоматизированных многопроцессорных информационных систем во всех сферах деятельности изменяет подходы и технологии, которые ранее в них применялись [2, с. 3]. Большое влияние эта тенденция оказала и на технологии организации учебно-воспитательного процесса.

С понятием «педагогическая диагностика» связано понятие «тестирование» как перспективный метод педагогической диагностики - направление на стыке педагогики, теории измерений, математических моделирования и статистики, автоматизации с использованием современных и перспективных средств вычислительной техники. Эта процедура контроля легко формализуется, следовательно, легко автоматизируется и реализуется в сетевых инфраструктурах. Тестирование является педагогическим явлением, имеющим многоплановое определение, сущность которого сводится к пониманию его как метода исследования и наиболее эффективного и объективного средства контроля, как часть современных и перспективных образовательных технологий [3, с. 79]. 
Концепции современного обучения рассматривают содержание образования как совокупность курсов, курс - как систему занятий, а занятие - как систему образовательных действий, каждое из которых, в свою очередь является совокупностью простых действий. Такая структура находит отражение в системе контроля знаний. При оценке качества знаний контролю подлежат не только их полнота, но и умение использовать их взаимосвязи, это можно сделать только в том случае, если предварительно составить структурную схему тестового задания и реализовать ее в современной сетевой инфраструктуре [4, с. 327], обозначить отдельные образовательные действия и их логические или причинно-следственные связи. Необходимо, чтобы тест проверял знания, умения и навыки обучаемого по реализации этих переходов с использованием современного высоко технологичного оборудования. Количество заданий по курсу достигает нескольких сотен и более, что свидетельствует о большой трудоемкости процесса создания тестов, а его функциональность может быть ограничена производительностью современных сетевых многопроцессорных комплексов [5, с. 161].

Выделяют показатели качества знаний, на которые контроль оказывает наибольшее влияние: соответствие полученных результатов поставленным целям; мотивация обучаемых; познавательная активность; производительность использования сетевых тестирующих систем. Рассматривая влияние системы контроля на повышение качества знаний, умений и навыков отмечают ее непосредственное влияние на данный критерий.

Большинство информационно образовательных ресурсов, содержащих тестовые материалы, можно разделить на две категории: ориентированные на прохождение студентами тестов в письменной форме с дальнейшей их проверкой (бланочное тестирование); автоматизированные системы тестирования с соответствующими программными компонентами [6, с. 105]. Положительные качества 
второй категории тестовых ресурсов очевидны. Именно в автоматизированных системах тестирования с сетевой архитектурой в наибольшей степени проявляются основные преимущества такого контроля знаний.

В инструментальном отношении тестирование является совокупностью последовательных педагогических действий, нацеленных на определение эффективности образовательной системы. Качественными характеристиками средств контроля являются: функциональное соответствие средств контроля дидактическим задачам, содержанию и избранным методам контроля в соответствии с требованиями стандарта и программы, потребностями и возможностями участников учебно-воспитательного процесса и эффективность сетевых компьютерных средств тестирования [7, с. 243].

Качественными характеристиками организации контроля являются: проведение и организация контрольных процедур в соответствии с требованиями программы и потребностями участников учебно-воспитательного процесса; реализация этих процедур в определенной психологически обстановке; вовлечение обучаемых в процесс проведения тестирующих процедур с целью формирования навыков самоконтроля; оптимальность и систематичность тестовых процедур; реализация этих процедур в определенной программно-аппаратной среде. Существует достаточное количество различных программ для тестирования, большую часть которых можно найти во «всемирной паутине». Однако некоторые из них является узконаправленными программами, созданными для поведения тестирования только по одному предмету. Многие тестирующие программы страдают отсутствием наглядности, в них нельзя задавать сложные формулы и иллюстрации что связано с особенностями их информационного обеспечения [8, с. 13]. Некоторые недостатки обработки, анализа, интерпретации и выдачи результатов тестирования обусловлены особенностями архитектуры и методики расчета производительности в таких инфраструктурах. 
В настоящее время наиболее распространены программы диагностики уровня подготовки студентов в виде тестов. Это самая легкая и простая форма программы, предполагающая следующую методику: вопрос - несколько вариантов ответа, один из которых правильный. Возможны вариации тестовых программ, когда каждому варианту ответа, в зависимости от степени его правильности или полноты присваивается весовой коэффициент, а конечная оценка выводится на основе анализа этих коэффициентов.

В связи с тем, что одной из основных задач контроля является развитие структур мышления, памяти, внимания, то применение автоматизированных многопроцессорных средств контроля, спроектированных или составленных в соответствии с основными принципами программного обеспечения таких систем [9, с. 13], будет способствовать переходу тестируемых на следующий уровень компетентности.

Оптимально разработанные тесты должны соответствовать не только предмету обучения, но и его задачам: готовится спецификация теста, в которой указываются тестируемые области, задачи обучения и тестирования, связи данного теста с другими и особенности каждой темы.

Функциональность тестовых заданий тесно связана с методикой обучения и особенностями программного обеспечения - текст не только сообщает знания, но и развивает мышление обучаемого. Эта концепция проверяется сложно формализуемыми вопросами на сравнение, обобщение, доказательство. Содержание заданий раскрывает понимание фактов, их связей и обобщений с использованием соответствующего программного обеспечения. Используется следующая последовательность возрастания сложности заданий: воспроизведение фактического материала; раскрытие причинно-следственных, временных и других связей; систематизация знаний.

Система автоматизированного педагогического контроля позволяет не только констатировать достигнутый уровень компетентно- 
сти, но и способствовать переходу обучаемых на следующий уровень: средства педагогического контроля проектируются на основе требований образовательного стандарта и программы обучения по предмету; средства контроля проектируются или составляются для нескольких уровней компетентности, при этом принимается во внимание возможность выбора заданий соответствующего уровня.

В качестве примера можно привести разработанную программную платформу VinEx, представляющую собой контролирующую систему, реализующую сетевую модель взаимодействия, работающую с базой ответов и вопросов $[10$, с. 60]. Они могут включать в себя графические изображения и аудиоинформацию, которые реализуются на многопроцессорных вычислительных системах. Результаты ответов анализируются в зависимости от заданной шкалы оценок, ответы проверяемого фиксируются, имеется возможность их просмотра. Опыт работы с данной контролирующей системой показал ее удобство, быстроту и эффективность оценки знаний, возможность работы в сетевой инфраструктуре учебного заведения [11, с. 7].

\section{Список литературы}

1. Бабешко В.Н., Набиуллина А.Р. Разработка средств компьютерного тестирования // Инновационная наука. 2015. № 11-2. С. 151-153.

2. Бабешко В.Н. Компьютерная оценка качества знаний // Новая наука: Проблемы и перспективы. Стерлитамак: РИЦ АМИ, 2016. № 4-1. С. 3-5.

3. Бабешко В.Н., Набиуллина А.Р. Компьютерная диагностика уровня знаний // Инновационная наука. 2015. № 8-1 (8). С. 79-80.

4. Бабешко В.Н. Распределенные информационно-вычислительные системы в туманных вычислительных сетях // Информационно-телекоммуникационные системы и технологии (ИТСИТ-2014): мат-лы Всероссийской науч.-практ. конф. Кемерово: КузГТУ, 2014. С. 327.

5. Бабешко В.Н., Бабешко С.В. Оценка производительности многопроцессорных вычислительных систем // Современные инстру- 
ментальные системы, информационные технологии и инновации. 2014. С. 161-164.

6. Бабешко В.Н. Информационное обеспечение тестирования // Приоритетные научные исследования и разработки. 2016. С. 105-107.

7. Бабешко В.Н., Набиуллина А.Р. Автоматизированный контроль качества обучения // Инновационная наука. 2015. № 9 (9). С. 243-245.

8. Бабешко В.Н., Бабешко С.В. Информационные компоненты современных вычислительных комплексов // Молодежь и системная модернизация страны: материалы междунар. научной конф. студентов и молодых ученых в 2-х томах. Курск: $3 \mathrm{AO}$ «Университетская книга», 2016. С. 13-16.

9. Бабешко С.В., Бабешко В.Н. Программное обеспечение многопроцессорных систем // Новая наука: Современное состояние и пути развития. Стерлитамак: РИЦ АМИ, 2016. № 4-3. С. 13-15.

10.Бабешко В.Н., Набиуллина А.Р. Технология тестового контроля уровня знаний // Инновационная наука. 2015. № 10-3. С. 60-62.

11. Бабешко В.Н. Средства автоматизированного контроля знаний // Новая наука: Теоретический и практический взгляд. Стерлитамак: РИЦ АМИ, 2016. № 4-2 (75). С. 7-9.

\section{References}

1. Babeshko V.N., Nabiullina A.R. Innovatsionnaya nauka. 2015. № 11-2, pp. 151-153.

2. Babeshko V.N. Novaya nauka: Problemy i perspektivy. Sterlitamak: RITs AMI, 2016. № 4-1, pp. 3-5.

3. Babeshko V.N., Nabiullina A.R. Innovatsionnaya nauka. 2015. № 8-1 (8), pp. 79-80.

4. Babeshko V.N. Informatsionno-telekommunikatsionnye sistemy i tekhnologii (ITSIT-2014): materialy Vserossiyskoy nauchno-prakticheskoy konf. [Information and Telecommunication Systems and Technologies (ITSIT 2014): Proceedings of the All-Russian Scientific and Practical Conference]. Kemerovo: KuzGTU, 2014. P. 327. 
5. Babeshko V.N., Babeshko S.V. Sovremennye instrumental'nye siste$m y$, informatsionnye tekhnologii $i$ innovatsii [Modern instrumented systems, information technology and innovation]. 2014, pp. 161-164.

6. Babeshko V.N. Prioritetnye nauchnye issledovaniya i razrabotki [Priority nye research and development]. 2016, pp. 105-107.

7. Babeshko V.N., Nabiullina A.R. Innovatsionnaya nauka. 2015. № 9 (9), pp. 243-245.

8. Babeshko V.N., Babeshko S.V. Molodezh'i sistemnaya modernizatsiya strany: materialy mezhdunar. nauchnoy konf. studentov i molo$d y k h$ uchenykh $v 2-k h$ to-makh [Youth and systemic modernization of the country: materials of the international scientific conference of students and young scientists]. Kursk: ZAO «Universitetskaya kniga», 2016, pp. 13-16.

9. Babeshko S.V., Babeshko V.N. Novaya nauka: Sovremennoe sostoyanie i puti razvitiya. Sterlitamak: RITs AMI, 2016. № 4-3, pp. 13-15.

10. Babeshko V.N., Nabiullina A.R. Innovatsionnaya nauka. 2015. № 103, pp. 60-62.

11. Babeshko V.N. Novaya nauka: Teoreticheskiy i prakticheskiy vzglyad. Sterlitamak: RITs AMI, 2016. № 4-2 (75), pp. 7-9.

\section{ДАННЫЕ ОБ АВТОРЕ}

Бабешко Владимир Николаевич, к.т.н., доцент

Новосибирский государственный университет экономики и управления

ул. Каменская 52/1, г. Новосибирск, 630099, Российская Федерачия

vnb67@mail.ru

\section{DATA ABOUT THE AUTHOR}

Babeshko Vladimir Nikolaevich, Ph.D., Associate Professor

Novosibirsk State University of Economics and Management 52/1, Kamenskaya Str., Novosibirsk, 630099, Russian Federation vnb67@mail.ru 